\title{
VEGFA rs3025020 Polymorphism Contributes to CALR- Mutation Susceptibility and Is Associated with Low Risk of Deep Vein Thrombosis in Primary Myelofibrosis
}

\author{
${ }^{1}$ Center for the Study of Myelofibrosis, Laboratory of Biochemistry, \\ Biotechnology and Advanced Diagnostics, Istituto di Ricovero e Cura \\ a Carattere Scientifico Policlinico S. Matteo Foundation, Pavia, Italy \\ 2 Laboratory of Biochemistry, Biotechnology and Advanced \\ Diagnostics, Istituto di Ricovero e Cura a Carattere Scientifico \\ Policlinico San Matteo Foundation, Pavia, Italy \\ 3 Biometry \& Clinical Epidemiology, Scientific Direction, Istituto di \\ Ricovero e Cura a Carattere Scientifico Policlinico San Matteo \\ Foundation, Pavia, Italy \\ ${ }^{4}$ Department of Immunology and Inflammation, Centre for \\ Haematology Research, Imperial College London, London SW7 2BU, \\ United Kingdom
}

Laura Villani ${ }^{1}$ Vittorio Rosti ${ }^{1}$ Margherita Massa ${ }^{2}$ Rita Campanelli $^{1}{ }^{10}$ Paolo Catarsi $^{1}$

Adriana Carolei ${ }^{1} \quad$ Carlotta Abbà $^{1} \quad$ Annalisa de Silvstri ${ }^{3}$ Robert Peter Gale ${ }^{4}$ Giovanni Barosi ${ }^{1}$ (1)

Address for correspondence Giovanni Barosi, MD, Center for the Study of Myelofibrosis, IRCCS Policlinico S. Matteo, Viale Golgi 19, 27100 Pavia, Italy (e-mail: barosig@smatteo.pv.it).

TH Open 2021;5:e513-e520.

\section{Abstract \\ Keywords \\ - primary myelofibrosis \\ - VEGFA polymorphism \\ - rs3025020 \\ - deep vein thrombosis \\ - CALR mutation}

Background Single nucleotide polymorphisms (SNPs) in vascular endothelial growth factor A (VEGFA) are associated with susceptibility to several diseases including cancer. Correlations between VEGFA rs3025020 genotypes with clinical and laboratory features of primary myelofibrosis (PMF) are unstudied.

Methods DNA was analyzed by real-time polymerase chain reaction for VEGFA rs3025020 genotypes in a cohort of 844 subjects with PMF and in two cohorts of normal subjects $(N=247$ and $N=107)$.

Results Frequency of rs3025020 minor allele ( $T$ ) was not significantly different in subjects with PMF compared with normals; however, the T-allele was more frequent in PMF subjects with a calreticulin (CALR)-mutated genotype compared with normals (35 vs. $27 \% ; \mathrm{OR}=1.47[95 \% \mathrm{Cl}, 1.09,1.98] p=0.011)$, especially in subjects with a CALRtype 2/type 2-like mutation (43 vs. $27 \%$; OR=2.01 $[1.25,3.24] p=0.004$ ). CALR mutants with the rs3025020 TT genotype had higher CXCR4 expression on CD34positive blood cells, and those who carried CT/TT genotypes had lower platelet concentrations compared with other genotypes at diagnosis. Overall, subjects with the rs3025020 CT/TT genotype had a lower cumulative incidence of deep vein thrombosis in typical sites (1.6 vs. $4.2 \%$; $\mathrm{OR}=0.37[0.15,0.90] p=0.029)$ and longer interval from diagnosis to first thrombosis ( $H R=0.37[0.14,0.95] p=0.039)$.

Conclusion Persons with PMF and the VEGFA rs3025020 minor T-allele are more likely to have a CALR mutation compared with other somatic driver mutations and lower cumulative incidence and hazard for deep vein thrombosis in typical sites. received

April 15, 2021

accepted after revision

July 15, 2021
DOI https://doi.org/

$10.1055 / \mathrm{s}-0041-1739293$.

ISSN 2512-9465. (c) 2021. The Author(s).

This is an open access article published by Thieme under the terms of the Creative Commons Attribution License, permitting unrestricted use, distribution, and reproduction so long as the original work is properly cited. (https://creativecommons.org/licenses/by/4.0/)

Georg Thieme Verlag KG, Rüdigerstraße 14, 70469 Stuttgart, Germany 


\section{Introduction}

Primary myelofibrosis (PMF) is a myeloproliferative disorder mostly caused by gain-of-function driver mutations in Janus kinase-2 (JAK2), calreticulin (CALR), or myeloproliferative leukemia virus (MPL). ${ }^{1}$ Persons with these mutations often have additional variations in ASXL1, EZH2, DMNT3A, IDH1, and IDH2. ${ }^{2}$ However, the mutation topography of PMF does not completely account for the different phenotypes including clinical and laboratory co-variates and risks of thrombosis, progression, and transformation to acute myeloid leukemia. Other gene loci are also important. For example, considerable data indicate some single nucleotide polymorphisms (SNPs) such as JAK2, MECOM, TERT, HBS1L-MYB, THRB-RARB, glucocorticoid receptor, and monocyte chemoattractant protein-1 predispose to developing PMF and/or correlate with clinical and prognostic features. ${ }^{3-11}$

Vascular endothelial growth factor A (VEGFA) is a proangiogenic protein correlated with the development and progression of myeloproliferative neoplasms, including PMF. ${ }^{12}$ SNPs in VEGFA are associated with susceptibility to several diseases, including cancer. ${ }^{13-15}$ We now report correlations between VEGFA rs3025020 genotypes and clinical and laboratory features of PMF.

\section{Materials and Methods}

\section{Study Population}

Stored DNA from blood granulocytes of 844 consecutive subjects with PMF, seen at the Center for the Study of Myelofibrosis of the IRCCS Policlinico S. Matteo Foundation in Pavia and included in a database, was the primary source material of this study. Clinical data were collected on the first visit and prospectively thereafter. Diagnosis was confirmed by reviewing the initial bone marrow biopsy and based on the WHO diagnostic criteria at the time of their first visit and re-classified according to 2017 revised WHO criteria. ${ }^{16}$ After the first examination, visits were scheduled every 6 months.

\section{Control Populations}

We used two control cohorts, one of healthy Italian participants in a bone marrow donor registry whose blood samples were anonymized $(N=247)$, and data generated from the public database 1000 Genomes Project_Phase 3_EUR_Subpopulation (Tuscans in Italy) $(N=107)$.

\section{SNP Analysis}

DNA was isolated from blood granulocytes, obtained by density gradient centrifugation, using the QIAamp DNA Blood Mini Kit (QIAGENSciences Inc. Germantown, Maryland, United States). SNP genotyping used a pre-designed, two-labeled (VIC-FAM) TaqMan Assay C_1647366_10 (Applied Biosystems, Foster City, California, United States). Reactions were done on a CFX96 Realtime PCR Detection System (Biorad Company, Hercules, California, United States) according to the manufacturer's instructions.

\section{Data Analyzed}

Data were collected at diagnosis and analyzed included age, spleen size by clinical measurement, CBC with differential, serum lactate dehydrogenase level and cholesterol concentration, percentage of blood blasts, and International Prognostic Scoring System (IPSS) risk category. ${ }^{17}$ In most subjects, blood concentrations of CD34-positive cells, ${ }^{18}$ and blood CXCR4 expression on CD34-positive cells were also quantified. ${ }^{19}$ Diagnoses of bone marrow biopsies were analyzed and fibrosis graded according to EUMNET consensus criteria. ${ }^{20}$ Cytogenetic analyses used standard techniques and were reported according to the International System for Human Cytogenetic Nomenclature criteria. ${ }^{21}$

$J A K 2^{V 617 \mathrm{~F}}$ and $M P L^{\mathrm{W} 515}$ were detected by the real-time polymerase chain reaction or high-resolution melting analyses. CALR mutations were identified by capillary electrophoresis and bi-directional sequencing. Next generation sequencing was used to detect mutations in selected myeloid neoplasm-related genes. Genomic and transcript analyses were performed using the diagnostic panel commercially available Oncomine Myeloid Research Assay (Thermofisher, Waltham, Massachusetts, United States). The genomic and transcript analysis were performed with IonReporter software applying the last release of Myeloid workflow (Thermofisher). Variations causing missense, frameshift, an altered stop/initiation codon, in-frame insertion/deletion or variants affecting splice site were regarded as mutations.

\section{Statistical Analyses}

Analyses considered clinical and laboratory co-variates at diagnosis. Continuous variables were presented as means $(+\mathrm{SD})$ or median with interquartile range (IQR). Categorical variables were expressed as percentages. Pearson $\chi$-test with one degree of freedom was used to compare allele and genotypes frequencies. Deviations from the Hardy-Weinberg equilibrium were tested by the Fisher exact test.

The independent contribution of $r s 3025020$ SNP to odds of PMF phenotypes was assessed by logistic regression analysis. Since this was an exploratory study, we did not introduce a correction for multiple comparisons. ${ }^{22}$ End points for associations with genotypes were blast transformation-free survival and survival. We further analyzed the outcome of major thromboses, including arterial thromboses (myocardial infarction, stroke), deep vein thrombosis and pulmonary embolism, and thrombosis in atypical sites (portal vein thrombosis, Budd-Chiari syndrome, and cerebral sinus thrombosis). Competing risk analysis was used to compute thrombosis cumulative incidence rates, considering overall mortality as the competing event. The Fine and Gray competing risks regression was used to study the determinants of thromboses. ${ }^{23}$ Subhazard ratio (sHR) and 95\% CI were computed. In this analysis, conventional risk factors measured at diagnosis, including age, sex, JAK2 ${ }^{\mathrm{V} 617 \mathrm{~F}}$ mutation, CALR mutation, white-blood cell count were analyzed. In multivariable regression, non-collinear variables associated with thrombosis with $p<0.1$ at univariable analysis, and with a frequency of missing values lower than $20 \%$, were used to assess whether VEGFA rs3025020 status 
Table 1 Baseline characteristics of subjects with primary myelofibrosis at diagnosis analyzed for rs3025020 VEGFA polymorphism

\begin{tabular}{|c|c|}
\hline & Primary myelofibrosis $(N=844)$ \\
\hline \multicolumn{2}{|l|}{ Demography } \\
\hline Age, y, median (IQR) & $52(40-61)$ \\
\hline Sex male, $N(\%)$ & $499(59.1)$ \\
\hline \multicolumn{2}{|l|}{ Clinical-hematological co-variates } \\
\hline Hemoglobin, g/L, mean ( $\pm S D)$ & $128(29)$ \\
\hline White blood cell count $\times 10 \mathrm{E}+9 / \mathrm{L}$, mean $( \pm \mathrm{SD})$ & $9.9(6.4)$ \\
\hline Platelet count $\times 10 \mathrm{E}+9 / \mathrm{L}$, mean $( \pm \mathrm{SD})$ & $510(347)$ \\
\hline Monocyte count $\times 10 \mathrm{E}+9 / \mathrm{L}$, mean $( \pm \mathrm{SD})$ & $606(507)$ \\
\hline Spleen size, $\mathrm{cm} \mathrm{E}+2$, mean $( \pm \mathrm{SD})$ & $148(99)$ \\
\hline IPSS score, low, $N(\%)$ & $515(61)$ \\
\hline IPSS score, intermediate- $1, N(\%)$ & $144(17)$ \\
\hline IPSS score, intermediate-2, N (\%) & $110(13)$ \\
\hline IPSS score, high, $N(\%)$ & $77(9)$ \\
\hline Plasma LDH, x ULN, mean ( \pm SD) & $1.64(1.13)$ \\
\hline Serum cholesterol, mg/dL, mean ( \pm SD) & $159(43)$ \\
\hline Blood CD34-positive cells $\times 10 \mathrm{E}+6 / \mathrm{L}$, mean $( \pm S D)$ & $59(160)$ \\
\hline $\begin{array}{l}\text { CXCR4 expression on blood CD34-positive } \\
\text { cells, \%, mean }( \pm S D)\end{array}$ & $42(25)$ \\
\hline \multicolumn{2}{|l|}{ Molecular characteristics } \\
\hline$J A K 2^{\mathrm{V} 617 \mathrm{~F}} N(\%)$ & $541(65.7)$ \\
\hline CALR mutation, $N(\%)$ & $171(20.7)$ \\
\hline MPL mutation, $N(\%)$ & $44(53.5)$ \\
\hline Triple negative, $N(\%)$ & $67(8.1)$ \\
\hline \multicolumn{2}{|l|}{ ASXL1 or EZH2 mutations, } \\
\hline - positive, $N(\%)$ & $45(18.7)$ \\
\hline - negative, $N(\%)$ & $195(81.3)$ \\
\hline \multicolumn{2}{|l|}{ Bone marrow histology (fibrosis) } \\
\hline Grade- $0, N(\%)$ & $259(30.8)$ \\
\hline Grade-1, N (\%) & $225(26.7)$ \\
\hline Grade-2, N (\%) & $241(28.6)$ \\
\hline Grade-3, N (\%) & $117(13.9)$ \\
\hline
\end{tabular}

Abbreviations: IQR, interquartile range; LDH, lactic dehydrogenase; SD, standard deviation; Triple negative, subjects without any of the myeloproliferative neoplasm driver mutations $\left(J A K 2^{\mathrm{V} 617 \mathrm{~F}}, C A L R, M P L^{\mathrm{W} 515}\right)$; ULN, upper limit of normal.

Note: Spleen size was measured using the spleen index calculated by multiplying the length of the longitudinal axis by the transverse axis. Monocyte concentration was available in 452 subjects; plasma LDH activity was available in 469 subjects; serum cholesterol concentration was available in 421 subjects; blood CD34-positive cell concentration was available in 398 subjects; CXCR4 expression on CD34-positive blood cells was available in 295 subjects.

independently predicted thrombotic occurrence. Results were considered statistically significant when two-sided $p$ values were less than 0.05 . These computations were performed with STATA 12 (Stata Corporation, College Station, Texas, United States).

All other computations were performed with STATISTICA software (Dell Technologies Inc. Round Rock, Texas, United States).

\section{Results}

Demographic and clinical co-variates of the 844 subjects with PMF are displayed in - Table 1. In total, 499 were male (59\%). Median age was 52 years (IQR, 40-61). Fifty-seven percent had a prefibrotic myelofibrosis. The IPSS risk distribution was low in $61 \%$, intermediate- 1 in $17 \%$, intermediate2 in $14 \%$, and high in $9 \%$ of the subjects. JAK2 ${ }^{\mathrm{V} 617 \mathrm{~F}}$ was 
Table 2 Genotype and allele frequencies of the rs3025020 polymorphism of VEGFA in 844 patients with primary myelofibrosis (PMF), 247 subjects of healthy control population, and 107 subjects reported from "Tuscan from Italy"

\begin{tabular}{|l|l|l|l|l|l|l|l|}
\hline & $N$ & \multicolumn{6}{|l|}{ rs3025020 polymorphism } \\
\hline & & CC & CT & TT & CC/CT & CT/TT & T allele frequency \\
\hline PMF subjects, $N$ (\%) & 844 & 407 & 354 & 83 & 761 & 437 & $520 / 1688$ \\
& & $(48.2)$ & $(41,9)$ & $(9.8)$ & $(90.2)$ & $(51.8)$ & $(30.8)$ \\
\hline Local healthy controls, $N(\%)$ & 247 & 134 & 92 & 21 & 226 & 113 & $134 / 494$ \\
& & $(54.3)$ & $(37.2)$ & $(8.5)$ & $(91.4)$ & $(45.7)$ & $(27.1)$ \\
\hline Tuscans from Italy, $N(\%)$ & 107 & 51 & 47 & 9 & 98 & 56 & $65 / 214$ \\
& & $(47.7)$ & $(43.9)$ & $(8.4)$ & $(91.6)$ & $(52.3)$ & $(30)$ \\
\hline
\end{tabular}

detected in $66 \%$ of subjects tested, $60 \%$ of whom had a heterozygous mutation, and $40 \%$ had a homozygous mutation. Thirty-nine of 136 subjects tested by cytogenetics (29\%) had an abnormal karyotype. A total of 72 subjects (14\%) had died after a median follow-up of 77 months (IQR, 36-150). Median survival is 21 years. Eighty-eight subjects (17\%) had blast transformation (BT) at a median of 26 years.

\section{Association with VEGFA rs3025020}

Genotype frequency distributions of VEGFA rs3025020 were consistent with the Hardy-Weinberg equilibrium in subjects with PMF and controls. Frequencies of the VEGFA rs3025020 alleles in PMF subjects did not differ significantly from local controls $(\mathrm{OR}=1.19[0.95,1.40] p=0.11)$, and "Tuscans in Italy" cohort $(\mathrm{OR}=1.02[0.75,1.39] p=0.89$; - Table 2).

\section{VEGFA rs3025020 SNP and PMF Somatic Driver Mutations}

PMF cohorts defined by the somatic driver mutations, i.e., $J A K 2^{\mathrm{V} 617 \mathrm{~F}}$, CALR, MPL mutants, or the so-called "triple negative" (no detectable driver mutation) were in Hardy-Weinberg equilibrium (-Supplementary Table S1). Compared with local healthy controls, rs3025020 T-allele variant was not enriched in subjects with $J A K 2^{\mathrm{V} 617 \mathrm{~F}}$ (28.8 vs. $27.1 \%$; $\mathrm{OR}=1.0[0.85,1.37] p=0.49)$. Conversely, in subjects without $J A K 2^{\mathrm{V} 617 \mathrm{~F}}$ the VEGFA rs3025020 T-allele was more frequent than in the local controls ( 34 vs. 27.1\%; OR, 1.39 [1.04, $1.80] p=0.015)$, and was higher than subjects with JAK2 $2^{\mathrm{V} 617 \mathrm{~F}}$ (37.2 vs. $28.8 \%$; $\mathrm{OR}=1.27[1.02,1.58] p=0.028$ ).

Subjects with a CALR mutation had a higher VEGFA rs3025020 T-allele frequency compared with local controls (35.4 vs. $27.1 \%$; OR $=1.47[1.09,1.98] p=0.011$ ), mostly because of an increased frequency in subjects with a CALRtype 2/type 2-like mutation compared with controls (42.8 vs. $27.1 \%$; OR $=2.01[1.25,3.24] p=0.004)$.

\section{VEGFA rs3025020 Genotype and PMF Co-variates}

Associations between VEGFA rs3025020 genotypes and PMF co-variates at diagnosis are displayed in - Supplementary Table S2. There were no significant correlations with age or sex. Subjects with the CT/TT genotype had a higher mean white blood cell (WBC) count compared with the CC genotype $(10.4$ vs. $9.5 \times 10 \mathrm{E}+9 / \mathrm{L} ; p=0.04)$. Subjects with the
VEGFA rs3025020 TT genotype had higher mean CXCR4 expression on CD34-positive cells compared with those with the CC/CT genotypes ( 54.4 vs. $40.8 \% ; p=0.005$ ). We detected no significant association between rs3025020 genotype and frequency of non-driver clonal mutations, cytogenetic abnormalities, or degree of bone marrow fibrosis at diagnosis.

We found inconsistent correlations between WBC or CXCR4 expression on CD34-positive cells and driver mutations in the whole cohort of PMF subjects. The association between VEGFA rs3025020 CT/TT genotype and higher mean WBC count was statistically significant only in $J A K 2^{\mathrm{V} 617 \mathrm{~F}}$ cohorts $\quad(11.5 \quad$ vs. $\quad 9.8 \times 10 \mathrm{E}+9 / \mathrm{L} ; \quad p=0.005$; -Supplementary Table S3), and was only significant in subjects with an allele burden $>50 \%$ (homozygous genotype) (14.7 vs. $11.3 \times 10 \mathrm{E} 9 / \mathrm{L} ; \quad p=0.002) \quad$ (-Supplementary Table S4). Higher CXCR4 expression on CD34-positive cells was significant in subjects with the rs3025020 TT genotype compared with the CT/CC genotype only in subjects without JAK2 ${ }^{\mathrm{V} 617 \mathrm{~F}}$ (59.9 VS. 23.7\%; $p=0.002 ;$ - Supplementary Table S5), and this association was detected only in subjects with a CALR mutation (-Supplementary Tables S6-S10 >).

We also detected an association between the VEGFA rs3025020 CT/TT genotype and a lower platelet concentration compared with the CC genotype in subjects with a CALR mutation (mean: 629 vs. $782 \times 10 \mathrm{E}+9 / \mathrm{L} ; \quad p=0.013$; -Supplementary Table 57). Associations of the VEGFA rs3025020 CT/TT genotype with a lower platelet concentration at diagnosis and the rs3025020 TT genotype with higher CXCR4 expression on CD34-positive cells were detected in type 1/type 1-like and type 2/type 2-like CALR mutation but were statistically significant in type 2/type 2-like CALR mutation (993 vs. $617 \times 10 \mathrm{E}+/ \mathrm{L} ; p=0.0031$; and 69 vs. 32\%; $p<0.001$; - Supplementary Table S8).

\section{VEGFA rs3025020 Genotype and Thrombosis}

In total, 168 subjects (20\%) had a major thrombotic event. Cumulative frequency of the first thrombotic event was 8.7, 10.4 and $15.9 \%$ in CARL- and MPL-mutated and triple negative individuals, respectively. On the contrary, subjects carrying the $J A K 2^{\mathrm{V} 617 \mathrm{~F}}$ mutation had the highest risk of thrombosis compared with all the other groups (25.2\%). $56.5 \%$ of the subjects had vein thrombosis in atypical sites, 
Table 3 Major thrombotic events stratified for rs3025020 VEGFA polymorphism

\begin{tabular}{|c|c|c|c|c|c|c|c|c|}
\hline & \multicolumn{6}{|c|}{ rs3025020 polymorphism } & \multirow{3}{*}{$\begin{array}{l}\text { CC vs. CT/TT } \\
\text { Odds ratio } \\
(95 \% \mathrm{CI})\end{array}$} & \multirow{3}{*}{$\begin{array}{l}\text { TT vs. } \mathrm{CC} / \mathrm{CT} \\
\text { Odds ratio } \\
(95 \% \mathrm{CI})\end{array}$} \\
\hline & All cases & CC & CT & TT & $\mathrm{CC} / \mathrm{CT}$ & $\mathrm{CT} / \mathrm{TT}$ & & \\
\hline & 843 & 406 & 354 & 83 & 760 & 437 & & \\
\hline $\begin{array}{l}\text { Overall thrombotic events, } N \\
\text { (\% of PMF cases) }\end{array}$ & $\begin{array}{l}168 \\
(19.9)\end{array}$ & $\begin{array}{l}90 \\
(22.1)\end{array}$ & $\begin{array}{l}66 \\
(18.6)\end{array}$ & $\begin{array}{l}12 \\
(14.4)\end{array}$ & $\begin{array}{l}156 \\
(20.5)\end{array}$ & $\begin{array}{l}78 \\
(17.8)\end{array}$ & $\begin{array}{l}0.76(0.54,1.07) \\
p=0.11\end{array}$ & $\begin{array}{l}0.65(0.34,1.23) \\
p=0.19\end{array}$ \\
\hline $\begin{array}{l}\text { Arterial thrombosis, } N \text { (\% of } \\
\text { PMF cases) }\end{array}$ & $\begin{array}{l}49 \\
(5.8)\end{array}$ & $\begin{array}{l}28 \\
(6.9)\end{array}$ & $\begin{array}{l}17 \\
(4.8)\end{array}$ & $\begin{array}{l}4 \\
(4.8)\end{array}$ & $\begin{array}{l}45 \\
(5.9)\end{array}$ & $\begin{array}{l}21 \\
(4.9\end{array}$ & $\begin{array}{l}0.68(0.38,1.22) \\
p=0.19\end{array}$ & $\begin{array}{l}0.80(0.28,2.29) \\
p=0.68\end{array}$ \\
\hline $\begin{array}{l}\text { - In year before diagnosis, } N \\
\text { (\% of thromboses) }\end{array}$ & $\begin{array}{l}14 \\
(28.6)\end{array}$ & $\begin{array}{l}7 \\
(25)\end{array}$ & $\begin{array}{l}7 \\
(41.2)\end{array}$ & $\begin{array}{l}0 \\
(0)\end{array}$ & $\begin{array}{l}14 \\
(31.1)\end{array}$ & $\begin{array}{l}7 \\
(33.3)\end{array}$ & & \\
\hline $\begin{array}{l}\text { - At diagnosis, } N \text { (\% of } \\
\text { thromboses) }\end{array}$ & $\begin{array}{l}12 \\
(24.5)\end{array}$ & $\begin{array}{l}9 \\
(32.1)\end{array}$ & $\begin{array}{l}2 \\
(11.8)\end{array}$ & $\begin{array}{l}1 \\
(25)\end{array}$ & $\begin{array}{l}11 \\
(24.4)\end{array}$ & $\begin{array}{l}3 \\
(14.3)\end{array}$ & & \\
\hline $\begin{array}{l}\text { - After diagnosis, } N \text { (\% of } \\
\text { thromboses) }\end{array}$ & $\begin{array}{l}23 \\
(46.9)\end{array}$ & $\begin{array}{l}12 \\
(42.8)\end{array}$ & $\begin{array}{l}8 \\
(47.1)\end{array}$ & $\begin{array}{l}3 \\
(75)\end{array}$ & $\begin{array}{l}20 \\
(44.4)\end{array}$ & $\begin{array}{l}11 \\
(52.4)\end{array}$ & & \\
\hline $\begin{array}{l}\text { Deep vein thrombosis in } \\
\text { typical sites, } N \text { (\% of PMF } \\
\text { cases) }\end{array}$ & $\begin{array}{l}24 \\
(2.8)\end{array}$ & $\begin{array}{l}17 \\
(4.2)\end{array}$ & $\begin{array}{l}7 \\
(1.9)\end{array}$ & $\begin{array}{l}0 \\
(0)\end{array}$ & $\begin{array}{l}24 \\
(3.1)\end{array}$ & $\begin{array}{l}7 \\
(1.6)\end{array}$ & $\begin{array}{l}0.37(0.15,0.91) \\
p=0,029\end{array}$ & $\begin{array}{l}0.18(0.01,2.98) \\
p=0.23\end{array}$ \\
\hline $\begin{array}{l}\text { - In year before diagnosis, } N \\
\text { (\% of thromboses) }\end{array}$ & $\begin{array}{l}3 \\
(12.5)\end{array}$ & $\begin{array}{l}2 \\
(11.8)\end{array}$ & $\begin{array}{l}1 \\
(14.3)\end{array}$ & $\begin{array}{l}0 \\
(0)\end{array}$ & $\begin{array}{l}3 \\
(12.5)\end{array}$ & $\begin{array}{l}1 \\
(14.3)\end{array}$ & & \\
\hline $\begin{array}{l}\text { - At diagnosis, } N \text { (\% of } \\
\text { thromboses) }\end{array}$ & $\begin{array}{l}5 \\
(28.8)\end{array}$ & $\begin{array}{l}3 \\
(17.6)\end{array}$ & $\begin{array}{l}2 \\
(28.6)\end{array}$ & $\begin{array}{l}0 \\
(0)\end{array}$ & $\begin{array}{l}5 \\
(20.8)\end{array}$ & $\begin{array}{l}2 \\
(28.6)\end{array}$ & & \\
\hline $\begin{array}{l}\text { - After diagnosis, } N \text { (\% of } \\
\text { thromboses) }\end{array}$ & $\begin{array}{l}16 \\
(66.6)\end{array}$ & $\begin{array}{l}12 \\
(70.6)\end{array}$ & $\begin{array}{l}4 \\
(23.5)\end{array}$ & $\begin{array}{l}0 \\
(0)\end{array}$ & $\begin{array}{l}16 \\
(66.6)\end{array}$ & $\begin{array}{l}4 \\
(57.1)\end{array}$ & & \\
\hline $\begin{array}{l}\text { Venous thrombosis in } \\
\text { atypical sites, } N \text { (\% of PMF } \\
\text { cases) }\end{array}$ & $\begin{array}{l}95 \\
(11.3)\end{array}$ & $\begin{array}{l}45 \\
(11.1)\end{array}$ & $\begin{array}{l}42 \\
(11.9)\end{array}$ & $\begin{array}{l}8 \\
(9.6)\end{array}$ & $\begin{array}{l}87 \\
(11.4)\end{array}$ & $\begin{array}{l}50 \\
(11.4)\end{array}$ & $\begin{array}{l}1.03(0.67,1.59) \\
p=0.86\end{array}$ & $\begin{array}{l}1.76(0.79,3.93) \\
p=0.16\end{array}$ \\
\hline $\begin{array}{l}\text { - In year before diagnosis, } N \\
\text { (\% of thromboses) }\end{array}$ & $\begin{array}{l}7 \\
(7.4)\end{array}$ & $\begin{array}{l}1 \\
(2.2)\end{array}$ & $\begin{array}{l}5 \\
(11.9)\end{array}$ & $\begin{array}{l}1 \\
(12.5)\end{array}$ & $\begin{array}{l}6 \\
(6.9)\end{array}$ & $\begin{array}{l}6 \\
(12)\end{array}$ & & \\
\hline $\begin{array}{l}\text { - At diagnosis, } N \text { (\% of } \\
\text { thromboses) }\end{array}$ & $\begin{array}{l}73 \\
(76.8)\end{array}$ & $\begin{array}{l}37 \\
(82.2)\end{array}$ & $\begin{array}{l}29 \\
(69)\end{array}$ & $\begin{array}{l}7 \\
(87.5)\end{array}$ & $\begin{array}{l}66 \\
(75.9)\end{array}$ & $\begin{array}{l}36 \\
(72)\end{array}$ & & \\
\hline $\begin{array}{l}\text { - After diagnosis, } N \text { (\% of } \\
\text { thromboses) }\end{array}$ & $\begin{array}{l}15 \\
(15.8)\end{array}$ & $\begin{array}{l}7 \\
(15.5)\end{array}$ & $\begin{array}{l}8 \\
(19)\end{array}$ & $\begin{array}{l}0 \\
(0)\end{array}$ & $\begin{array}{l}15 \\
(17.2)\end{array}$ & $\begin{array}{l}8 \\
(16)\end{array}$ & & \\
\hline
\end{tabular}

Abbreviation: PMF, primary myelofibrosis.

77\% of which were synchronous with PMF diagnosis (-Table 3). Twenty-nine percent of the subjects had arterial thrombosis and $14.3 \%$ had deep vein thrombosis in typical sites. The frequency of thrombotic events was lower in subjects with the VEGFA rs3025020 CT/TT genotypes compared with those with the VEGFA rs3025020 CC genotype (17.8 vs. $22.1 \%$; OR, $0.76[0.54,1.07] p=0.117)$. A lower frequency of thrombotic events in subjects with the VEGFA rs3025020 CT/TT genotype was detected in the three thrombosis categories but significant only in the deep vein thromboses in typical site category $(\mathrm{OR}=0.37[0.15$, $0.90] p=0.029)$. Subjects with the rs3025020 TT or CT genotype $(N=437)$ developed seven events $(1.6 \%)$, with a post-diagnosis cumulative event rate of 0.21 per 100 person-years compared with a VEGFA rs3025020 CC genotype $(N=406)$ who had 17 events $(4.2 \%)$ with a post-diagnosis cumulative event rate of 0.60 per 100 person-years $(p=0.025)$.

With the KM analysis, the VEGFA rs3025020 genotype was significantly associated with the risk of incurring into deep vein thromboses in typical sites; it resulted higher in patients who had CC genotype with respect to those with $\mathrm{CT} / \mathrm{TT}$ genotype $(\mathrm{HR}=0.37[0.14,0.95] p=0.038)$. With the Fine and Gray model, with thrombotic event of interest and overall mortality of the competing risk, the cumulative incidence of deep vein thrombosis in typical sites at the end of follow-up was 6.7\% (95\% CI, 3.1, 12.2) (-Fig. 1). When refining the assessment of VEGFA rs3025020 status for the risk of thrombosis, we showed an increase in risk for CC genotype with respect to CT/TT genotype, with sHR for CT/TT $0.37(0.14,0.95) p=0.039$. At the time of thrombosis, $17 \%$ of patients were under cytoreductive therapy (90\% hydroxyurea). Cytoreductive therapy use was not a prognostic factor of longer thrombosis free survival in the Cox proportional hazards model. When the sHR of thrombotic events was adjusted for the risk factors for thrombosis, the outcome resulted independently predicted by the VEGFA rs3025020 CC genotype and by older age at the initial assessment (-Table 4). 


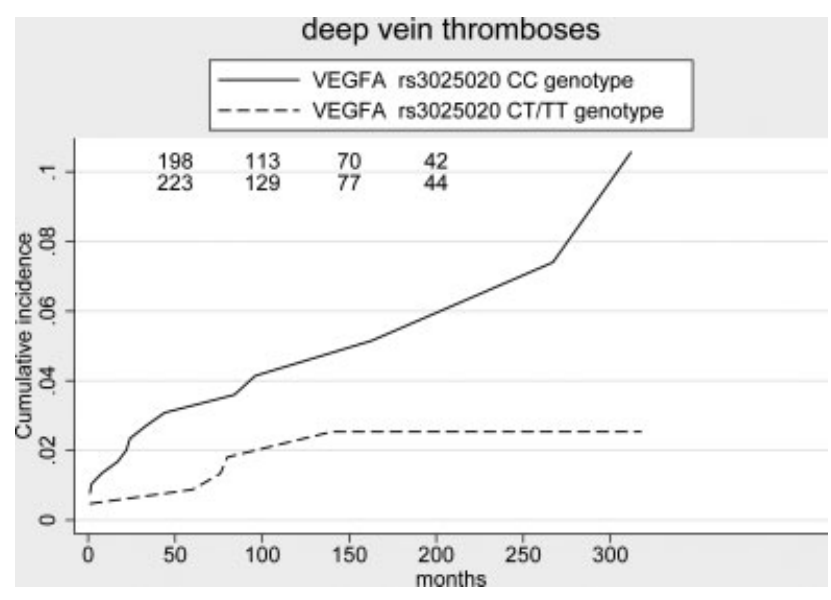

Fig. 1 Cumulative incidence of deep vein thrombosis in typical sites in 844 subjects with PMF stratified for the rs3025020 VEGFA polymorphism genotypes: subjects with CT/TT genotype had a significant lower risk of thrombosis $(p=0.039)$.

\section{VEGFA rs3025020 Genotypes and Survival}

We detected no significant correlations between VEGFA rs3025020 genotypes and survival or risk of BT (HR, 1.02 $[0.78,1.34] p=0.88 ; \mathrm{HR}=1.14[0.82,1.56] p=0.42)$.

\section{Discussion}

Our study indicates that persons with PMF with a CALR mutation, especially type 2/type 2-like, have an increased frequency of T-allele VEGFA rs3025020 genotypes. This association is in keeping with data of other SNPs in persons with MPNs. Trifa et al reported associations of TERT rs2736100 and MECOM rs2201862 genotypes with CALRmutated MPNs. ${ }^{7}$ The TERT rs 2736100 correlation was independent of molecular subtype, whereas the MECOM rs2201862 T-allele genotype was restricted to patients with type-1/type-1-like mutations. Lighezan et al reported associations between TET2 rs1548483 genotype and

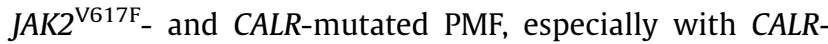
type 2 mutations. ${ }^{24}$

The imbalance of $r s 3025020$ frequency in CALR-mutated PMF may arise directly from an effect(s) of the VEGFA polymorphism or linkage to other gene(s). We found subjects with a CALR mutation with the VEGFA rs3025020 T-allele genotype having lower platelet concentrations at diagnosis than other VEGFA rs3025020 genotypes, and those with the TT genotype having higher CXCR4 expression on CD34positive cells compared with other genotypes. These correlations were strongest in subjects with CALR- type 2/type 2like mutations.

In CALR-mutated PMF persons, thrombocytosis is the dominant phenotype reflecting myeloproliferation; in PMF, reduced CXCR4 expression on CD34-positive cells is associated with disease activity and poor prognosis. ${ }^{19}$ This implies that the VEGFA rs3025020 T-allele acts directly, or through tight linkage to another gene(s), to regulate myeloproliferation/disease activity in this setting. The cor-
Table 4 Multivariate proportional hazards regression: predictive factors for deep vein thrombosis in typical sites

\begin{tabular}{|l|l|l|l|}
\hline Parameter & $\begin{array}{l}\text { Hazard } \\
\text { ratio }\end{array}$ & $\begin{array}{l}95 \% \\
\text { confidence } \\
\text { interval }\end{array}$ & $p$-Value \\
\hline Age at diagnosis $>65 \mathrm{y}$ & 3.08 & $1.21,7.82$ & 0.018 \\
\hline rs3025020 CT/TT & 0.38 & $0.15,0.96$ & 0.042 \\
\hline CALR-mutated & 0.55 & $0.13,2.28$ & 0.411 \\
\hline WBC count $>12 \times 10 \mathrm{E}+9 / \mathrm{L}$ & 1.31 & $0.51,3.41$ & 0.577 \\
\hline JAK2 ${ }^{\mathrm{V} 17 F}$-positive & 0.86 & $0.29,2.52$ & 0.782 \\
\hline Sex, male & 0.75 & $0.30,1.86$ & 0.537 \\
\hline
\end{tabular}

Abbreviations: CALR, calreticulin; WBC, white blood cells.

relations we found in CALR-mutated persons were poorly consistent across other driver mutations: in persons with homozygous $J A K 2^{\mathrm{V} 617 \mathrm{~F}}$ mutations we found association of the CT/TT genotype with a higher WBC concentration at diagnosis. The mechanism(s) underlying this requires further study.

The most interesting and clinically-important correlation we detected was the association between rs3025020 and thrombosis risk. The high incidence of thrombosis in our subjects allowed us to distinguish between arterial and deep vein thromboses in typical and atypical sites. Subjects with a rs3025020 T-allele genotype had a lower cumulative incidence and hazard of thromboses, particularly deep vein thrombosis in typical sites. Current knowledge about the potential impact of the VEGFA rs3025020 on angiogenesis and endothelial function is limited and the mechanism(s) unknown.

The low frequency of deep vein thrombosis in our series did not allow to dissect whether the different PMF driver clonal mutations had influence on the risk of thrombosis. However, the coincidence of high susceptibility to CALR mutation and low risk of thrombosis in subjects bearing the T-allele of rs3025020 VEGFA polymorphism claims for a hypothetical explanation of the low risk of thrombosis in CALR-mutated PMF reported in literature ${ }^{25}$ (we confirmed in this study).

Our study has limitations. First, it was retrospective, limiting the validity of interpreting some results, especially comparisons of rates and hazards of thromboses. Second, not adjusting for multiple comparison might result in false positives. However, the results of this study clearly illustrate and prove the concept that the constitutional genetic background is an important determinant of the risk of thrombosis in PMF.

In conclusion, we found that persons with PMF and VEGFA rs3025020 minor T-allele genotypes are more likely to have a CALR driver mutation compared with other driver mutations and a lower cumulative incidence and hazard for deep vein thrombosis in typical sites. If validated there may be clinical and therapy implications. 


\section{What Is Known on This Topic?}

- Vascular endothelial growth factor A (VEGFA) is a special type of cytokine, which is secreted by various cells such as vascular endothelial cells, and plays an important role in regulating blood vessel formation, tumor growth and development and atherosclerosis. VEGFA is known to exert a major influence on the development and progression of primary myelofibrosis (PMF).

- The human VEGFA gene is located on chromosome 6p21.3, whose single nucleotide polymorphisms (SNPs) can affect gene expression by altering key regulatory sequences or by altering mRNA stability at key regulatory loci.

- rs3025020 VEGFA gene polymorphism is a predisposing polymorphism for many diseases including malignancy. No documentation of increased susceptibility to vascular disease has been reported. Correlations between VEGFA rs3025020 genotypes with clinical and laboratory features of PMF are unstudied.

\section{What Does This Paper Add?}

- Subjects with PMF who carry the minor T-allele of the rs3025020 VEGFA polymorphism emerged as enriched in the CALR-mutated PMF.

- CALR-mutated subjects who carry VEGFA rs3025020 CT/TT genotypes displayed lower platelet count and those carrying TT genotype displayed higher expression of CXCR4 on CD34-positive cells, both markers of myeloproliferation/disease activity.

- Individuals who carry the T-allele of VEGFA rs3025020 had an overall lower risk of deep vein thrombosis in typical sites.

- Higher susceptibility of the VEGFA rs3025020 T-allele in CALR-mutated PMF and lower risk of thrombosis in individuals carrying the $\mathrm{T}$-allele provide a hypothetical explanation of the known low-thrombotic risk in CALR-mutated PMF.

\section{Informed Consent}

Subjects gave written informed consent approved by the IRCCS Policlinico S. Matteo Foundation Institutional Ethics Committee to be included in the database. The Ethics Committee of the Hospital approved a separate written informed consent for subjects to donate blood and bone marrow samples for molecular research on their disease.

\section{Funding}

The study is supported by AIRC $5 \times 1000$ call "Metastatic disease: the key unmet need in oncology" to MYNERVA project, \#21267 (MYeloid Research Venture AIRC) and Ricerca Corrente IRCCS Policlinico San Matteo Foundation,
Pavia, Italy, project number 874 , code number 08054517 , received by Vittorio Rosti (www.sanmatteo.org).

Conflict of Interest

None declared.

\section{References}

1 Rumi E, Trotti C, Vanni D, Casetti IC, Pietra D, Sant'Antonio E. The genetic basis of primary myelofibrosis and its clinical relevance. Int J Mol Sci 2020;21(23):8885

2 Grinfeld J, Nangalia J, Baxter EJ, et al. Classification and personalized prognosis in myeloproliferative neoplasms. N Engl J Med 2018;379(15):1416-1430

3 Jones AV, Chase A, Silver RT, et al. JAK2 haplotype is a major risk factor for the development of myeloproliferative neoplasms. Nat Genet 2009;41(04):446-449

4 Olcaydu D, Harutyunyan A, Jäger R, et al. A common JAK2 haplotype confers susceptibility to myeloproliferative neoplasms. Nat Genet 2009;41(04):450-454

5 Kilpivaara O, Mukherjee S, Schram AM, et al. A germline JAK2 SNP is associated with predisposition to the development of JAK2 (V617F)-positive myeloproliferative neoplasms. Nat Genet 2009; 41(04):455-459

6 Tapper W, Jones AV, Kralovics R, et al. Genetic variation at MECOM, TERT, JAK2 and HBS1L-MYB predisposes to myeloproliferative neoplasms. Nat Commun 2015;6:6691

7 Trifa AP, Bănescu C, Bojan AS, et al. MECOM, HBS1L-MYB, THRBRARB, JAK2, and TERT polymorphisms defining the genetic predisposition to myeloproliferative neoplasms: a study on 939 patients. Am J Hematol 2018;93(01):100-106

8 Lighezan DL, Bojan AS, Iancu M, et al. TET2 rs1548483 SNP associating with susceptibility to molecularly annotated polycythemia vera and primary myelofibrosis. J Pers Med 2020;10(04): 259

9 Tefferi A, Lasho TL, Mudireddy M, et al. The germline JAK2 GGCC (46/1) haplotype and survival among 414 molecularly-annotated patients with primary myelofibrosis. Am J Hematol 2019;94(03): 299-305

10 Poletto V, Rosti V, Villani L, et al. A3669G polymorphism of glucocorticoid receptor is a susceptibility allele for primary myelofibrosis and contributes to phenotypic diversity and blast transformation. Blood 2012;120(15):3112-3117

11 Masselli E, Carubbi C, Cambò B, et al. The -2518 A/G polymorphism of the monocyte chemoattractant protein-1 as a candidate genetic predisposition factor for secondary myelofibrosis and biomarker of disease severity. Leukemia 2018;32(10):2266-2270

12 Gadomska G, Stankowska K, Boinska J, et al. VEGF-A, sVEGFR-1, and sVEGFR-2 in BCR-ABL negative myeloproliferative neoplasms. Medicina (Kaunas) 2017;53(01):34-39

13 Ferrara N. Pathways mediating VEGF-independent tumor angiogenesis. Cytokine Growth Factor Rev 2010;21(01):21-26

14 Metzger CS, Koutsimpelas D, Brieger J. Transcriptional regulation of the VEGF gene in dependence of individual genomic variations. Cytokine 2015;76(02):519-526

15 Eng L, Azad AK, Habbous S, et al. Vascular endothelial growth factor pathway polymorphisms as prognostic and pharmacogenetic factors in cancer: a systematic review and meta-analysis. Clin Cancer Res 2012;18(17):4526-4537

16 Swerdow SH, Campo E, Harris NL, et al, eds. WHO Classification of Tumours of Haematopoietic and Lymphoid Tissues. Revised 4th ed. IARC: Lyon; 2017

17 Cervantes F, Dupriez B, Pereira A, et al. New prognostic scoring system for primary myelofibrosis based on a study of the International Working Group for Myelofibrosis Research and Treatment. Blood 2009;113(13):2895-2901 
18 Barosi G, Viarengo G, Pecci A, et al. Diagnostic and clinical relevance of the number of circulating CD34 $(+)$ cells in myelofibrosis with myeloid metaplasia. Blood 2001;98(12):3249-3255

19 Barosi G, Rosti V, Catarsi P, et al. Reduced CXCR4-expression on CD34-positive blood cells predicts outcomes of persons with primary myelofibrosis. Leukemia 2021;35(02):468-475

20 Thiele J, Kvasnicka HM. Myelofibrosis-what's in a name? Consensus on definition and EUMNET grading. Pathobiology 2007;74 (02):89-96

21 Larson DP, Akkari YM, Van Dyke DL, et al. Conventional cytogenetic analysis of hematologic neoplasms: a 20-year review of proficiency test results from the College of American Pathologists/American College of Medical Genetics and Genomics Cytogenetics Committee. Arch Pathol Lab Med 2021;145(02):176-190
22 Streiner DL, Norman GR. Correction for multiple testing: is there a resolution? Chest 2011;140(01):16-18

23 Fine JP, Gray RJ. A proportional hazards model for the subdistribution of a competing risk. J Am Stat Assoc 1999; 94:496-509

24 Trifa AP, Bănescu C, Tevet M, et al. TERT rs2736100 A>C SNP and JAK2 46/1 haplotype significantly contribute to the occurrence of JAK2 V617F and CALR mutated myeloproliferative neoplasms-a multicentric study on 529 patients. Br J Haematol 2016;174(02): 218-226

25 Finazzi MC, Carobbio A, Cervantes F, et al. CALR mutation, MPL mutation and triple negativity identify patients with the lowest vascular risk in primary myelofibrosis. Leukemia 2015;29(05): $1209-1210$ 